\title{
Two-Loop Corrections to the Leptonic Decays of Quarkonium
}

\author{
M. Beneke, ${ }^{1}$ A. Signer, ${ }^{1}$ V. A. Smirnov ${ }^{2}$ \\ ${ }^{1}$ Theory Division, CERN, CH-1211 Geneva 23, Switzerland \\ ${ }^{2}$ Nuclear Physics Institute, Moscow State University, 119889 Moscow, Russia
}

(Received 8 December 1997)

\begin{abstract}
Applying asymptotic expansions at threshold, we compute the two-loop quantum chromodynamics (QCD) correction to the short-distance coefficient that governs the leptonic decay $\psi \rightarrow l^{+} l^{-}$of an $S$-wave quarkonium state and discuss its impact on the relation between the quarkonium nonrelativistic wave function at the origin and the quarkonium decay constant in full QCD. [S0031-9007(98)05640-3]
\end{abstract}

PACS numbers: $13.20 . \mathrm{Gd}, 12.38 . \mathrm{Bx}$

Quarkonium decays played an important role in establishing quantum chromodynamics (QCD) as a weakly interacting theory at short distances. Calculations of heavy quarkonium decays usually proceed under the assumption that the heavy quark-antiquark bound state is nonrelativistic and that the decay amplitude factorizes into the bound state wave function at the origin and a shortdistance quark-antiquark annihilation amplitude. One can then explain the small width of the $S$-wave spin-triplet charmonium state $J / \psi$, because it can decay only through electromagnetic annihilation or annihilation into at least three gluons [1]. Today's understanding of quarkonium bound states has refined this picture and allows us to calculate relativistic corrections systematically at the expense of introducing further nonperturbative parameters that characterize the bound state. Such calculations can be done most transparently in the framework of a nonrelativistic effective field theory (NRQCD) [2,3] that implements the factorization of contributions to the (partial) decay widths from different length scales. Besides potentially large relativistic corrections, the size of radiative corrections to the quark-antiquark annihilation amplitudes has always been a matter of concern. The one-loop radiative corrections to the decays $\psi \rightarrow l^{+} l^{-}$(where $\left.l=e, \mu\right)$ [4], $\psi \rightarrow$ light hadrons, and $\psi \rightarrow \gamma+$ light hadrons [5] are large and question the practicability of factorization for charmonium and, perhaps, even bottomonium. [Here and in the following we use $\psi$ as a label for any $S$-wave spin-triplet state, i.e., $J / \psi$ or $\psi^{\prime}$ for charmonium and $\Upsilon(n S)$ for bottomonium.]

In this Letter we address this question and report on the calculation of two-loop short-distance corrections to the leptonic decay $\psi \rightarrow l^{+} l^{-}$. This is the first (and also "easiest") two-loop matching calculation for quarkonium decays, and it can also be used to connect the decay constant of the $\psi$ meson defined in QCD with the nonrelativistic wave function at the origin, which appears in NRQCD and potential models. In turn, this wave function is an important input parameter for the prediction of other quarkonium decays and also quarkonium production cross sections.

We recall that $\psi$ decays leptonically through interaction with the electromagnetic current. The partial decay rate, neglecting the tiny lepton masses, is given exactly by

$$
\Gamma\left(\psi \rightarrow l^{+} l^{-}\right)=\frac{4 \pi e_{Q}^{2} \alpha_{\mathrm{em}}^{2} f_{\psi}^{2}}{3 M_{\psi}},
$$

where $M_{\psi}$ is the mass of $\psi, \alpha_{\mathrm{em}}$ the fine structure constant, and $e_{Q}$ the electric charge of the heavy quark in units of the electron charge. The $\psi$ decay constant $f_{\psi}$ is defined through the following matrix element of the electromagnetic current:

$$
\left\langle\psi(p)\left|\bar{Q} \gamma_{\mu} Q\right| 0\right\rangle=(-i) f_{\psi} M_{\psi} \epsilon_{\mu}^{*}(p) .
$$

$\left[\epsilon_{\mu}(p)\right.$ is the $\psi$ polarization vector and $p$ the $\psi$ momentum.] The leptonic decay rates are known experimentally [6]. For $J / \psi$ we find $f_{J / \psi}=(405 \pm 15) \mathrm{MeV}$.

The decay constant parametrizes the strong interaction effects and contains long- and short-distance contributions. For quarkonium the short-distance scale is $1 / M_{\psi}$ and the long-distance (bound state) scales are $1 /\left(M_{\psi} v\right)$ and $1 /\left(M_{\psi} v^{2}\right)$, where $v$ is the (small) characteristic velocity of the $\psi$ 's quark constituents. The short-distance contributions can be isolated, and calculated in perturbation theory, by matching the vector current in QCD onto a series of operators in NRQCD. Up to corrections of order $v^{4}$, the matching relation is given by

$$
\left\langle\psi(p)\left|\bar{Q} \gamma^{\mu} Q\right| 0\right\rangle=\Lambda^{\mu i}(p)\left[C_{0}\left(\alpha_{s}, \frac{m_{Q}}{\mu}\right)\left\langle\psi\left|\psi^{\dagger} \sigma_{i} \chi\right| 0\right\rangle(\mu)+\frac{C_{1}\left(\alpha_{s}, \frac{m_{Q}}{\mu}\right)}{6 m_{Q}^{2}}\left\langle\psi\left|\psi^{\dagger} \vec{D}^{2} \sigma_{i} \chi\right| 0\right\rangle(\mu)+\mathcal{O}\left(v^{4}\right)\right]
$$

where $\psi$ (not to be confused with the $\psi$ meson) and $\chi$ denote nonrelativistic two spinors, $\vec{D}$ is the spatial covariant derivative, and $m_{Q}$ is the heavy quark mass.
(More details on notation and NRQCD can be found in Ref. [3]. Note, however, that we use a relativistic normalization of states also for the matrix elements in 
NRQCD.) The matrix elements on the right-hand side are defined in the $\psi$ rest frame, and $\Lambda(p)$ is the matrix that performs the Lorentz boost into this frame. The matching coefficients $C_{0}$ and $C_{1}$ are expressed as series in the strong coupling $\alpha_{s}$ and account for the shortdistance QCD corrections. The matching coefficients and matrix elements in NRQCD individually depend on the factorization scale $\mu . C_{1}$ is defined (as in Ref. [7]) such that $C_{1}=1+\mathcal{O}\left(\alpha_{s}\right)$ and [4]

$$
\begin{aligned}
C_{0}\left(\alpha_{s}, \frac{m_{Q}}{\mu}\right)= & 1-\frac{2 C_{F} \alpha_{s}\left(m_{Q}\right)}{\pi} \\
& +c_{2}\left(m_{Q} / \mu\right)\left(\frac{\alpha_{s}}{\pi}\right)^{2}+\ldots,
\end{aligned}
$$

where $C_{F}=\left(N_{c}^{2}-1\right) /\left(2 N_{c}\right)$, and $N_{c}=3$ is the number of colors. We now discuss the calculation of the two-loop matching coefficient $c_{2}$.

Since the matching coefficient contains only shortdistance effects, it can be obtained by replacing the quarkonium state $\psi$ on both sides of Eq. (3) by a free quark-antiquark pair of on-shell quarks at small relative velocity. In terms of this on-shell matrix element, the matching equation can be rewritten as

$$
Z_{2, \mathrm{QCD}} \Gamma_{\mathrm{QCD}}=C_{0} Z_{2, \mathrm{NRQCD}} Z_{J}^{-1} \Gamma_{\mathrm{NRQCD}}+\mathcal{O}\left(v^{2}\right),
$$

where $Z_{2}$ are the on-shell wave function renormalization constants in QCD and NRQCD, and $\Gamma$ is the amputated, bare electromagnetic annihilation vertices in QCD and NRQCD. The Feynman diagrams for $\Gamma_{\mathrm{QCD}}$ at two loops are shown in Fig. 1. Since the current $J=\psi^{\dagger} \sigma_{i} \chi$ need not be conserved in NRQCD, we allowed for its renormalization, $J_{\text {bare }}=Z_{J} J_{\text {ren }}$, on the right-hand side. We then obtain $C_{0}$ by calculating all other quantities in Eq. (5) in dimensional regularization and using the modified minimal subtraction scheme ( $\overline{\mathrm{MS}}$ scheme) [8].
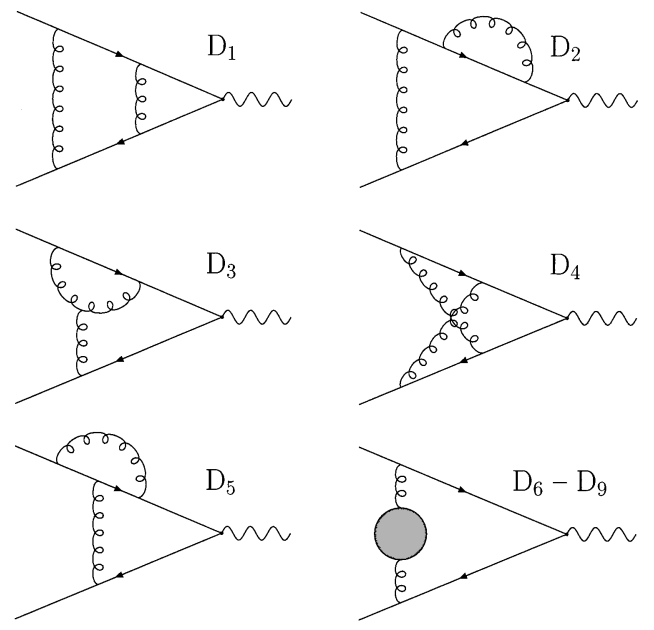

FIG. 1. Diagrams that contribute to $\Gamma_{\mathrm{QCD}}$. Symmetric diagrams exist for $D_{2,3,5}$. The last diagram summarizes vacuum polarization contributions from massless fermions $\left(D_{6}\right)$, gluons $\left(D_{7}\right)$, ghosts $\left(D_{8}\right)$, and the massive fermion with mass $m_{Q}\left(D_{9}\right)$.
The matching calculation is considerably simplified, if one uses the threshold expansion of Ref. [9] to compute $\Gamma_{\mathrm{QCD}}$ directly as an expansion in $v^{2}$. The threshold expansion is obtained by writing contributions corresponding to hard $\left(l \sim m_{Q}\right)$, soft $\left(l \sim m_{Q} v\right)$, potential $\left(l_{0} \sim m_{Q} v^{2}, l_{i} \sim m_{Q} v\right)$, and ultrasoft $\left(l \sim m_{Q} v^{2}\right)$ regions. The contributions from soft, potential, and ultrasoft loop momenta can all be identified with diagrams in NRQCD that appear in the calculation of $\Gamma_{\text {NRQCD. }}$. Hence, they drop out of the matching relation Eq. (5) and it suffices to compute the contribution to the threshold expansion of the diagrams in Fig. 1, where all loop momenta are hard. (The threshold expansion is not only convenient; it also provides an implicit definition of NRQCD in dimensional regularization. This is necessary, because dimensionally regularized NRQCD is not given by the dimensionally regularized Feynman integrals constructed from the vertices and propagators of NRQCD. In order to avoid that the cutoff for the effective theory be treated as larger than $m_{Q}$, dimensionally regularized NRQCD has to be supplemented by a prescription for expanding the Feynman integrands. This prescription is provided to all orders as part of the diagrammatic threshold expansion method [9].)

We briefly describe the calculation of the hard contributions to $\Gamma_{\mathrm{QCD}}$. (Details of the calculational method and a solution to the recurrence algorithm for the two-loop integrals will be given in a long write-up of this Letter.) The spinor structure of the on-shell matrix element in QCD is conventionally parametrized by two form factors, $F_{1}$ and $F_{2}$, of which only the combination $F_{1}+F_{2}$ is required here. Since terms of order $v^{2}$ are not needed to determine $C_{0}$ [see Eq. (5)], we may set the relative momentum to zero and compute the form factors directly at threshold. The form factors have Coulomb singularities at threshold and diverge as $1 / v^{2}$. However, these singularities appear only in the soft, potential, and ultrasoft contributions, and the hard contribution is well defined directly at threshold in dimensional regularization. The loop integrals simplify considerably, once the relative momentum is set to zero, since they then depend only on a single scale. We then project on the form factor $F_{1}+F_{2}$ and reduce all integrals to integrals without numerators. These integrals can be further reduced to "simple" integrals and two nontrivial two-loop integrals by means of recurrence relations derived from integration by parts in the loop momenta [10]. The solution to the recurrence relations is the difficult part of the calculation. The remaining nontrivial two-loop integrals can be calculated explicitly using standard Feynman parameters.

After summing all the diagrams, multiplying by the two-loop QCD on-shell wave function renormalization constant [11], and performing (one-loop) coupling and mass renormalization, the result still contains poles in $\epsilon=$ $(4-d) / 2$ (where $d$ is the space-time dimension). Since the wave function renormalization constant $Z_{2, \mathrm{NRQCD}}$ in 
NRQCD equals 1 up to higher-order terms in $v^{2}$, not needed here, these poles are attributed to an anomalous dimension of $J$, which first arises at the two-loop order. As a consequence the matrix element $\left\langle\psi\left|\psi^{\dagger} \sigma_{i} \chi\right| 0\right\rangle(\mu)$ is factorization scale dependent. We define it in the $\overline{\mathrm{MS}}$ scheme and obtain the anomalous dimension for the NRQCD vector current $J$ :

$\gamma_{J}=\frac{\mathrm{d} \ln Z_{J}}{\mathrm{~d} \ln \mu}=-C_{F}\left(2 C_{F}+3 C_{A}\right) \frac{\pi^{2}}{6}\left(\frac{\alpha_{s}}{\pi}\right)^{2}+\mathcal{O}\left(\alpha_{s}^{3}\right)$.

The scale dependence is compensated by the scale dependence of the two-loop matching coefficient $c_{2}\left(m_{Q} / \mu\right)$ in Eq. (4). Separating the different color group factors $C_{F}=4 / 3, C_{A}=3, T_{F}=1 / 2$, the final result for $c_{2}\left(m_{Q} / \mu\right)$ in the $\overline{\mathrm{MS}}$ scheme is

$$
\begin{aligned}
c_{2}\left(m_{Q} / \mu\right)= & C_{F}^{2} c_{2, A}+C_{F} C_{A} c_{2, N A}+C_{F} T_{F} n_{f} c_{2, L} \\
& +C_{F} T_{F} c_{2, H}, \\
c_{2, A}= & \pi^{2}\left[\frac{1}{6} \ln \left(\frac{m_{Q}^{2}}{\mu^{2}}\right)-\frac{79}{36}+\ln 2\right] \\
& +\frac{23}{8}-\frac{\zeta(3)}{2}, \\
c_{2, N A}= & \pi^{2}\left[\frac{1}{4} \ln \left(\frac{m_{Q}^{2}}{\mu^{2}}\right)+\frac{89}{144}-\frac{5}{6} \ln 2\right] \\
& -\frac{151}{72}-\frac{13 \zeta(3)}{4},
\end{aligned}
$$

$$
f_{\psi}=\left(1-\frac{8 \alpha_{s}\left(m_{Q}\right)}{3 \pi}-\left(44.55-0.41 n_{f}\right)\left(\frac{\alpha_{s}}{\pi}\right)^{2}+\ldots\right) f_{\psi}^{\mathrm{NR}}\left(m_{Q}\right) .
$$

With $\alpha_{s}\left(m_{c}\right) \approx 0.35$ and $\alpha_{s}\left(m_{b}\right) \approx 0.21$, the secondorder correction exceeds the first-order correction even for the bottomonium states. For charmonium, the secondorder term is almost twice as large as the already sizable first-order correction. [Note that the Brodsky-LepageMackenzie (BLM) estimate of the two-loop correction $[7,14,17]$ is far off the exact two-loop result.] Perturbative matching at the scale $\mu=m_{Q}$ does not seem to work. Can the factorization of short- and long-distance effects still be useful?

A novel, and perhaps unexpected, aspect at the two-loop level is the factorization scale dependence of the nonrelativistic decay constant and, hence, the quarkonium wave function at the origin. The scale dependence is large, especially due to the non-Abelian term in Eq. (6). This scale dependence of the wave function indicates the limitation of the nonrelativistic potential model approach already at leading order in $v^{2}$, since the wave functions obtained from solving the Schrödinger equation are scale independent. Despite this shortcoming it may be argued that the wave function/nonrelativistic decay constant obtained from potential models corresponds - if to anything - to the wave function evaluated at a scale typical for the bound state and

$$
\begin{gathered}
c_{2, L}=\frac{11}{18}, \\
c_{2, H}=-\frac{2 \pi^{2}}{9}+\frac{22}{9} .
\end{gathered}
$$

Here $\zeta(3)=1.202 \ldots$, and we have taken all fermions with masses less than $m_{Q}$ as massless, which is a good approximation even for $m_{Q}=m_{b}$, the bottom quark mass, in which case we neglect $m_{c}$, the charm quark mass. The massive (light) quark vacuum polarization contributions $c_{2, H}\left(c_{2, L}\right)$ agree with earlier calculations in Refs. [12,13] and $[7,13,14]$, respectively. Note also that the $C_{F}^{2}$ term of the form factors $F_{1,2}$ close to threshold has been calculated by Hoang [15], using the absorptive parts of the form factors obtained in Ref. [16]. Because the result in Ref. [15] contains hard and soft (potential, ultrasoft) contributions, it is not possible to extract the matching coefficient $c_{2, A}$ from Ref. [15]. (The structures in $c_{2, A}$ that cannot arise from the small-loop momentum regions agree with Hoang's result.)

The size of the two-loop correction to $C_{0}$ [Eq. (4)] given by Eqs. (7)-(11) is enormous. We define the (scale-dependent) nonrelativistic decay constant as

$$
\left\langle\psi\left|\psi^{\dagger} \sigma_{i} \chi\right| 0\right\rangle(\mu)=(-i) f_{\psi}^{\mathrm{NR}}(\mu) M_{\psi} \epsilon_{i}^{*},
$$

in analogy with Eq. (2). The nonrelativistic decay constant is related to the $\psi$ wave function at the origin by $M_{\psi}\left(f_{\psi}^{\mathrm{NR}}\right)^{2}=12|\Psi(0)|^{2}$. Using Eq. (3), we obtain

not $m_{Q}$. This point of view is also evident if the wave function is computed nonperturbatively using lattice NRQCD, in which case the ultraviolet cutoff/factorization scale is also much smaller than $m_{Q}$. We consider $\mu=1 \mathrm{GeV}$ as an adequate bound state scale for bottomonium and charmonium, as the applicability of perturbation theory prevents us from taking yet smaller scales. We then find, for bottomonium $\left(m_{b}=5 \mathrm{GeV}, n_{f}=4\right)$,

$$
\begin{aligned}
f_{Y(n S)}= & {\left[1-\frac{8 \alpha_{s}\left(m_{b}\right)}{3 \pi}-1.74\left(\frac{\alpha_{s}\left(m_{b}\right)}{\pi}\right)^{2}+\ldots\right] } \\
& \times f_{\Upsilon(n S)}^{\mathrm{NR}}(1 \mathrm{GeV}) \\
\approx & 0.81 f_{\Upsilon(n S)}^{\mathrm{NR}}(1 \mathrm{GeV}) .
\end{aligned}
$$

The numerical factor 0.81 is stable against variations of the scale of the coupling at fixed factorization scale $1 \mathrm{GeV}$. At this factorization scale the second-order correction is numerically insignificant. Although we do not know whether the three-loop correction would also be small at the low factorization scale, we tend to consider Eq. (14) as an accurate prediction. This prediction could be tested, if $f_{\mathrm{YR}(n S)}^{\mathrm{NR}}(1 \mathrm{GeV})$ were accurately known, for example, from NRQCD lattice simulations. The large scale dependence 
raises the theoretical question (the answer to which we postpone to the long write-up) as to whether it is necessary to resum the logarithms $\ln \left(m_{Q}^{2} / \mu^{2}\right)$ to all orders.

The situation is less favorable for charmonium states. Since $m_{c} \approx 1.5 \mathrm{GeV}$, the size of the second-order correction is altered little for scales $\mu>1 \mathrm{GeV}$. We have not succeeded in finding a trustworthy interpretation of Eq. (13) and conclude that the factorization of shortdistance and long-distance effects may not be useful in practice for charmonium. Since the leptonic decay is the simplest conceivable decay, this puts into question the possibility of obtaining universal relations between various charmonium decays and production processes through the use of NRQCD $[3,18]$. This pessimistic conclusion may be biased by our use of the $\overline{\mathrm{MS}}$ factorization scheme. It is conceivable that other factorization schemes or relations between physical observables, from which the wave function at the origin is eliminated, exhibit better convergence properties of their perturbative series. A definitive conclusion on this issue can be obtained only once a second quarkonium decay is computed to two-loop order.

We thank G. Buchalla for useful discussions and A. Hoang for correspondence. V.S. has been supported by the Russian Foundation for Basic Research, Project No. 96-01-00726, and by INTAS, Grant No. 93-0744.

Note added.-While this paper was being written, Czarnecki and Melnikov [19] also considered the twoloop matching of the electromagnetic current, also using the asymptotic expansion method of [9]. After correction of a trivial normalization error [20], their result agrees with ours.

[1] T. Appelquist and H. D. Politzer, Phys. Rev. Lett. 34, 43 (1975); A. De Rújula and S. L. Glashow, Phys. Rev. Lett. 34, 46 (1975).
[2] W. E. Caswell and G. P. Lepage, Phys. Lett. 167B, 437 (1986).

[3] G. T. Bodwin, E. Braaten, and G. P. Lepage, Phys. Rev. D 51, 1125 (1995); 55, 5853(E) (1997).

[4] R. Barbieri, R. Gatto, R. Körgerler, and Z. Kunszt, Phys. Lett. 57B, 455 (1975). The one-loop result can also be inferred from earlier QED calculations [R. Karplus and A. Klein, Phys. Rev. 87, 848 (1952)].

[5] P. B. Mackenzie and G. P. Lepage, Phys. Rev. Lett. 47, 1244 (1981)].

[6] Particle Data Group, R. M. Barnett et al., Phys. Rev. D 54, 1 (1996).

[7] E. Braaten and Y.-Q. Chen, OHSTPY-HEP-T-18 (hep-ph/ 9710357).

[8] W. A. Bardeen, A. J. Buras, D. W. Duke, and T. Muta, Phys. Rev. D 18, 3998 (1978).

[9] M. Beneke and V.A. Smirnov, CERN-97-315 (hep-ph/ 9711391).

[10] K. G. Chetyrkin and F. V. Tkachov, Nucl. Phys. B192, 159 (1981).

[11] D. J. Broadhurst, N. Gray, and K. Schilcher, Z. Phys. C 52, 111 (1991).

[12] S. G. Karshenboim, Yad. Fiz. 56, N12, 155 (1993) [Phys. At. Nucl. 56, 1710 (1993)].

[13] A. H. Hoang, J.H. Kühn, and T. Teubner, Nucl. Phys. B452, 173 (1995).

[14] M. B. Voloshin, Int. J. Mod. Phys. A 10, 2865 (1995).

[15] A. H. Hoang, Phys. Rev. D 56, 7276 (1997).

[16] R. Barbieri, J. A. Mignaco, and E. Remiddi, Nuovo Cim. 11A, 824 (1972).

[17] S. J. Brodsky, A. H. Hoang, J.H. Kühn, and T. Teubner, Phys. Lett. B 359, 355 (1995).

[18] For reviews, see E. Braaten, S. Fleming, and T.C. Yuan, Annu. Rev. Nucl. Part. Sci. 46, 197 (1996); M. Beneke, "The Strong Interaction, from Hadrons to Partons," Stanford, 1996, p. 549 (hep-ph/9703429).

[19] A. Czarnecki and K. Melnikov, Preceding Letter, Phys. Rev. Lett. 80, 2531 (1998).

[20] A. Czarnecki and K. Melnikov (private communication). 